AUTORES:

Fabio Antônio Tenório de Melo

Rogério Wagner da Silva

Bianca Alvarenga R Galletti

Camila da Silva B Evangelista

Patrícia Pollyana R Oliveira

Jonathan Campos Sousa Leite

https://doi.org/10.5628/rpcd.17.S5A.84
Efeitos do treinamento

com plataforma vibratória

na composição corporal

\section{em adultos jovens}

\section{sedentários}

PALAVRAS CHAVE:

Vibração. Exercício.

Percepção subjetiva de esforço.

Treinamento.
Training effects with vibrating platform on body

composition in sedentary young adults

\section{ABSTRACT}

The aim of this study was to analyze the anthropometric variation in exercise with vibration in sedentary adults. The sample consisted of 26 subjects divided into two groups: vibration (VG) and ergometry (EG), both evaluated regarding body composition. The VG was subjected to 15-minute sessions on the vibrating platform, once a day, three times a week for six weeks. EG was subjected to 20-minute sessions in an aerobike, with one daily session three times per week for six weeks. After the intervention, it was not possible to notice statistically significant changes in body composition in EG and VG groups. These results may be due to the little intervention time (six weeks), the lack of food control and the level of exercise intensity. EG's intensity aerobic exercise varied between 4 and 6 in the subjective perception of effort (SPE) evaluated with an adapted version of the Borg Scale, and the heart rate (HR) varied between $65-75 \%$ of MHR; these values can be considered moderated in line of other adopted protocols. After the intervention period the groups continued to be monitored for a period of six weeks, returning to physical inactivity to evaluate the effects of detraining

\section{KEYWORDS:}

Vibration. Exercise.

Effort subjective perception. Training. GE e GV. Tais resultados podem ser advindos possivelmente do pouco tempo de intervenção (seis semanas), da ausência do controle alimentar e o nível da intensidade dos exercícios. 0 exercício aeróbio adotado pelo GE teve a intensidade adotada entre 4 e 6 na percepção subjetiva de esforço (PSE), em escala adaptada de Borg, e a frequência cardíaca (FC) entre $65-75 \%$ da FCM, valores considerados moderados conforme protocolos adotados. Depois do período de intervenção os grupos continuaram a ser monitorados por um período de six semanas, retornando a inatividade física para avaliação dos efeitos do destreinamento.

O objetivo desse estudo foi analisar a variação antropométrica no exercício com vibração em grupos: Vibração (GV) e Ergometria (GE), ambos avaliados na composição corporal. 0 GV fo submetido a sessões de 15 minutos na plataforma vibratória, uma vez por dia, três vezes na semana por seis semanas. 0 GE foi submetido a sessões de 20 minutos na aerobike, com uma sessão diária três vezes por semana, durante seis semanas. Após a intervenção, não foi 
Há pouco tempo tem se tornado comum a utilização do treinamento de vibração de corpo inteiro por seus benefícios em um curto período de tempo por sessão. Esta ferramenta capaz de estimular fibras musculares produzindo um grande número de contrações, colaborando para a saúde do organismo. Estudos têm apontado o treino em plataforma vibratória para perda de peso, redução da massa de gordura, aumento da massa muscular, melhora na densidade mineral óssea e diminuição na circunferência de cintura. Programas de treinamento como aeróbio e de força, por exemplo, são oferecidos visando aumentar à adesão a atividade física, que além de eficaz na perda de peso tem mostrado bons resultados em aumentar e manter a massa muscular, estimular o metabolismo, ganho de força dentre outros benefícios (Capra, Tartaro, Magalhães, \& Martelli, 2016).

0 exercício aeróbio refere-se aos exercícios em que os grande músculos do corpo se movem de uma forma rítmica e durante períodos prolongados, se realiza sobretudo a custo do metabolismo aeróbio, ou seja com a presença de oxigênio, é conhecido por melhorar as condições cardiorrespiratórias, tônus muscular e mudanças na composição corporal (Haskell, Lee, \& Pate, 2007; Huang et al., 2014; Liu, Kenneth, \& Jasminka, 2011; de Oliveira Belmiro \& Navarro, 2016; Osawa \& Oguma, 2013; Rocha \& Snitynski, 2016; Silva \& Nunes, 2015).

Mediante o importante papel que a atividade física tem na prevenção de doenças, organizações de saúde como o American College of Sports Medicine e a American Heart Association recomendam a prática regular de atividade física. Várias modalidades são ofertadas atualmente, dentre elas uma nova tecnologia vem se destacando, a plataforma vibratória. A vibração, que é gerada pela plataforma, pode ser compreendida como movimento alternado de um corpo sólido em junção ao seu centro de equilíbrio; ou ainda, como um deslocamento oscilatório que se repete em torno de uma posição de referência (Alkahtani, King, Hills, \& Byrne, 2013; Hallal, Marques, \& Gonçalves, 2010; Silva \& Schneider, 2011). A plataforma vibratória é uma intervenção eficiente na prevenção de quedas em idosos. No que se refere a estímulos mecânicos de baixa amplitude e alta frequência é um método eficaz para melhorar a força muscular, equilíbrio corporal e a capacidade da mobilidade funcional, pode ser captada como movimento alternado de um corpo sólido em relação ao seu sistema ósseo, fazendo que melhore a densidade mineral óssea (DMO). Treinamentos utilizando plataforma estão se tornando mais populares como um método seguro e eficaz para melhoria da força muscular (Santiago et al., 2015; Silva \& Nunes, 2015).

Diante disto, o objetivo desse estudo foi analisar a variação antropométrica no exercício em plataforma vibratória em indivíduos adultos jovens sedentários.

\section{AMOSTRA}

Este estudo clínico randomizado foi realizado com adultos jovens e saudáveis, que decidiram participar voluntariamente, foram convidados a através de mensagem enviada para todos os alunos pela intranet da universidade. Critérios de inclusão: ser maior de 18 anos de idade, ser sedentário (sem prática de atividade física regular por no mínimo 60 dias antes do início do procedimento de intervenção) e assinar o Termo de Livre Consentimento Esclarecido (TLCE). Critérios de exclusão: apresentar condições que pudessem sugerir limitações as sessões de intervenção em plataforma vibratória, tais como: incapacidade física temporária; gestantes ou lactantes, existência de traumas osteoarticulares e/ou ausência superior a $25 \%$ nas sessões de treinamento. 0 estudo foi submetido ao Comitê de Ética da Universidade Católica de Brasília (UCB), teve parecer com aprovação favorável em 12/05/2015 sob número 020386/2015, em consonância com a resolução no 466/12 do Conselho Nacional de Saúde (CNS) que normatiza as pesquisas com seres humanos no Brasil. Inicialmente 45 voluntários foram selecionados, onde foram realizadas as orientações iniciais e familiarização com os protocolos e equipamentos de treinamento. A coleta dos dados ocorreu entre Setembro de 2015 de Dezembro de 2015

\section{INSTRUMENTOS}

Avalição da composição corporal

Foi utilizado absortometria radiológica de dupla energia (DXA), marca Lunar, modelo DPX-IQ (software versão 4.7e) no modo médio (velocidade de amostragem $5 \mathrm{~mm} / \mathrm{s}$ e corrente da ampola de RX $750 \mathrm{~mA}$ ); a temperatura da sala de avaliação durante as medidas foi mantida entre 18ㅇ e 25ㅇ; todos os ajustes em conformidade com as orientações contidas no manual do equipamento. 0 peso corporal total $(\mathrm{kg})$ e altura $(\mathrm{m})$ foram medidos descalços e com roupas adequadas usando balança eletrônica (Filizola ${ }^{\circledR}$ ) para a medição de peso corporal e um estadiômetro (SECA ${ }^{\oplus} 214$, EUA) foi utilizado para medir a altura. 0 valor do índice de massa corporal (IMC) foi calculado a partir da proporção de peso do corpo em quilogramas pelo quadrado da altura em metros obtida $\left(\mathrm{kg} / \mathrm{m}^{-2}\right)$. A massa livre de gordura e massa gorda foi determinada pelo DXA.

Avaliação Hematológica

Foi realizada por citometria de fluxo fluorescente e impedância utilizando o "XE2100-Sysmex" a contagem celular foi totalmente automatizada com alto padrão de qualidade adotado.

Bicicletas Ergométricas

Foram utilizadas duas bicicletas da marca/modelo AeroBike - $R 6$ total exercise que estimulam simultaneamente os membros superiores e inferiores. 0 treino era iniciado com carga de treino na Posição 02, que aplica $10.31 \mathrm{Kgf}$ para os membros superiores e $9.31 \mathrm{kgf}$ para os membros inferiores. 
Plataformas Vibratórias

Foram utilizadas duas plataformas vibratórias modelo Power Plate ${ }^{\circledR}$ Pro6 Air e um goniômetro da marca Cardiomed ${ }^{\oplus}$ para ajustar o angulo correto da flexão dos joelhos.

PROCEDIMENTOS

Protocolo de Treinamento

Os participantes foram separados aleatoriamente em dois grupos: Grupo Plataforma Vibratória (GV) e Grupo Ergometria (GE). Antes do início dos programas de treinamento, os participantes realizaram procedimento de familiarização nos equipamentos utilizados durante o estudo.

O GE foi submetido a sessões de atividade aeróbia em ciclo ergômetro durante 20 minutos, com 01 sessão diária 03 vezes por semana, durante 06 semanas. Durante as sessões de treino, o aumento da carga nas bicicletas era ajustado continuamente de acordo com a percepção subjetiva de esforço relatada na escala de Borg adaptada (Júnior, Prestes, Leite, \& Rodriguez, 2011)

Para garantir que a intensidade estivesse sempre na faixa pretendida para realizar o treinamento os pesquisadores apresentavam aos voluntários a escala e eles indicavam sua sensação de esforço a cada dois minutos, se a percepção relatada fosse inferior a classificação 03 a carga da ergometria era aumentada, se o relato fosse superior a classificação 06 a carga era reduzida.

O GV foi submetido a sessões de estimulação em plataforma vibratória, de acordo com o programa de treinamento elaborado pelos próprios pesquisadores com base nos estudos de Moezy, Olyaei, Hadian, Razi e Faghihzadeh (2008), que foi constituído de 15 minutos, com 01 sessão diária 03 vezes por semana, durante 06 semanas. Durante o estudo as frequências utilizadas nas plataformas foram alteradas a cada duas semanas $(30 \mathrm{~Hz}, 35 \mathrm{~Hz}$ e $40 \mathrm{~Hz})$ e a amplitude utilizada era alternada semanalmente $(2 \mathrm{~mm}$ e $4 \mathrm{~mm})$ a descrição detalhada da periodização do treinamento realizada pode ser observada no Anexo 01 . Como durante o treinamento vibratório a frequência e amplitude não eram alteradas durante a sessão de treino o questionamento sobre a percepção subjetiva de esforço somente era apresentada imediatamente após o término do treino. Antes do início da sessão de treino cada indivíduo realizou um aquecimento prévio de cinco minutos, em bicicleta estacionária e sem resistência. Foi adotada uma postura inicial estática durante as sessões de vibração, os participantes permaneceram em pé sobre a plataforma com os joelhos semi-flexionados, para assegurar maior ativação muscular foi adotada uma angulação de $30^{\circ}$ graus na articulação do joelho. A posição de estimulação foi padronizada, colocando o plano sagital do corpo sobre o ponto marcado no centro da base da plataforma vibratória e alinhando os pés à mesma distância dos ombros, descalços ou utilizando somente meias, com os membros superiores estendidos ao longo do tronco segurando em alças laterais que transmitem a vibração também para os membros superiores. Enquanto o posicionamento dinâmico se iniciava partindo do posicionamento estático e realizando os movimentos previstos no programa de treinamento. Ao final de cada treino os indivíduos eram questionados sobre percepção subjetiva de esforço relatada na escala de Borg adaptada (Júnior et al., 2011).
0 volume de trabalho de ambos os grupos durante o período de intervenção foi de 18 sessões de treinamento totalizando 6 horas de treinamento, foi adotado intervalo mínimo de 24 horas e máximo de 72 horas, entre as sessões de treinamento. As sessões de treinamento foram agendadas e realizadas sempre no mesmo horário, com intuito de atenuar os vieses hormonais advindos das variações circadianas. 0 acompanhamento das sessões de treinamento foi monitorado sempre pelos mesmos professores de educação física a fim de garantir o cumprimento do protocolo de intervenção e assegurar a segurança e a integridade física dos participantes do grupo GV e GE. Não houve nenhuma intervenção na rotina nutricional.

Após o período de intervenção todos os indivíduos continuaram sendo acompanhados por igual período, mas nesta segunda fase sem a estimulação física para avaliação dos efeitos do destreinamento. Todos voluntários foram monitorados semanalmente através de um aplicativo de celular para assegurar que tinham voltado a sua rotina anterior de sedentarismo e que não realizassem nenhuma atividade física nesta fase e retornassem a sua rotina anterior.

ANÁLISE ESTATÍSTICA

Após uma verificação exploratória com as medidas descritivas de média e desvio padrão, as análises foram realizadas no software SSPS, versão 23.0. Com a finalidade de testar a normalidade na distribuição dos dados, foi aplicado o teste de Kolmogorov Smirnov e Shapiro Wilks. Para verificar alterações intragrupo e intergrupos (GV e GE) e a análise dos dados adotando os momentos de coleta definidos em 03 tempos (T1, T2 e T3), foi utilizada análise de variância (Anova Split Plot). 0 índice de variância - covariância dos grupos foi realizada através do teste Wilk's Lambda. Para avaliar os efeitos intrasujeitos nas variáveis dependentes, anteriormente descritas, considerando a análise isolada com relação ao tempo, intensidade, e combinada tempo/intensidade, foi usado o teste de esfericidade Greenhouse-Geisser. 0 ajustamento das múltiplas comparações foi feito através do teste de Bonferroni. Em todas as análises foi adotado o nível de significância $p \leq .05$.

\section{RESULTADOS}

Após a realização do protocolo de familiarização os integrantes do GV relataram desconforto durante as sessões dinâmicas mesmo com uma percepção subjetiva de esforço menor quando comparada ao GE. Somente uma voluntária do GV relatou dores lombares ao final do treinamento. A organização do estudo é ilustrada no fluxograma a seguir descrevendo os procedimentos de seleção, alocação, design e perda amostral total. 


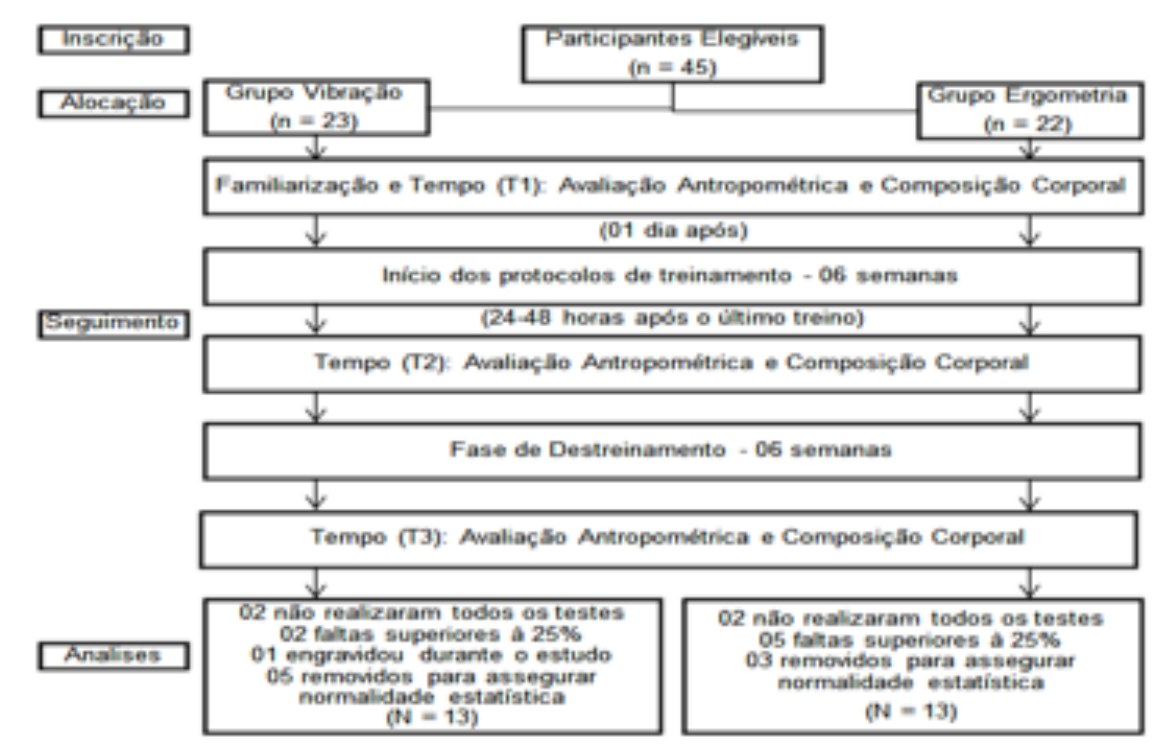

FIGURA 1. Fluxograma descritivo dos procedimentos do estudo.

0 quadro 1 apresenta as características gerais da amostra nos dois grupos. Os resultados são apresentados com média e desvio-padrão para cada uma das informações apresentadas. Estatisticamente os grupos são normais e não apresentam diferenças estatísticas nas medidas descritivas entre eles.

QUADRO 1. Composição corporal e antropométrica de voluntários.

\begin{tabular}{lcc}
\hline CARACTERÍSTICA & GV & GE \\
\hline Idade $($ anos $)$ & $20.77 \pm 2.86$ & $20.62 \pm 4.35$ \\
\hline Peso $(\mathrm{kg})$ & $58.77 \pm 9.16$ & $57.73 \pm 13.84$ \\
\hline Altura $(\mathrm{m})$ & $1.61 \pm 0.05$ & $1.64 \pm 0.06$ \\
\hline Índice de Massa Corporal $\left(\mathrm{kg} \cdot \mathrm{m}-{ }^{2}\right)$ & $22.72 \pm 3.67$ & $21.31 \pm 4.18$ \\
\hline Massa Magra $(\mathrm{kg})$ & $40.37 \pm 3.31$ & $40.26 \pm 5.78$ \\
\hline Massa Gorda $(\mathrm{kg})$ & $18.36 \pm 7.16$ & $17.47 \pm 8.69$ \\
\hline
\end{tabular}

Estatisticamente os grupos apresentam distribuição normal e não diferem significativamente entre eles ( $p$ >.05)

Em relação às variáveis antropométricas, os resultados não apontaram diferenças significativas nos itens peso corporal total entre os grupos $(p>.05)$, mas observa-se que nos momentos T1(Pós) e T2 (Destreinamento) que em ambos os grupos, houve uma certa inclinação à redução do peso corporal.
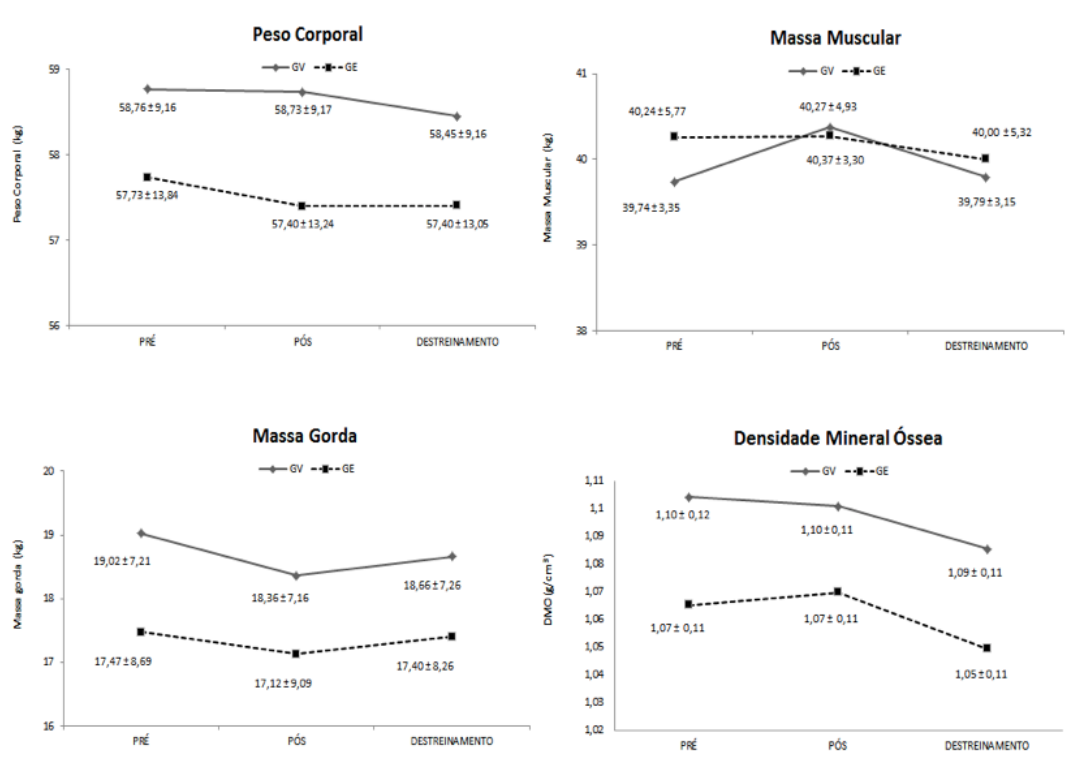

FIGURA 2. Peso Corporal, Massa Muscular, Massa Gorda e Densidade Mineral Óssea

Estatisticamente as variáveis estudadas não apresentaram diferenç̧as significativas $(p>050)$

Conforme análise realizada na variável massa muscular, o GV apresentou uma propensão maior ao seu ganho quando comparado ao GE, contudo os resultados também, não apresentaram significância estatística durante as análises $(p>.05)$. Mas apesar disso, clinicamente observou-se um incremento de aproximadamente $500 \mathrm{~g}$ de massa muscular em seis semanas de exercício no grupo GV. Pensando de forma linear é possível inferir que, prolongando esse período por aproximadamente 27 semanas, seria possível estimar um aumento da massa muscular de mais ou menos $2.170 \mathrm{~kg}$ a esse grupo. Porém é sabido que o corpo sofre adaptações ao treinamento, por conseguinte, seriam necessários ajustes em suas cargas para que os ganhos e as metas estabelecidas fossem mantidos.

Já na variável massa gorda, ambos os grupos apresentaram comportamentos similares, com um declínio maior para o GV após as 06 semanas de treinamento. Após o período do destreinamento os voluntários do GV ainda se mantiveram com o total aferido abaixo da avaliação inicial, sugerindo um possível prolongamento nas alterações desta variável. Contudo só podemos sugerir haver uma propensão positiva para estes efeitos, já que, conforme as análises estatísticas, estes não mostraram significância ( $p>.05)$.

Ao avaliar a densidade mineral óssea (DMO), também foi possível perceber que não houve diferença significativa entre os grupos. Porém quando analisamos somente os tempos $(1,2,3)$ existe uma tendência que estes valores se tornes significativos, pois os resultados não apontam uma diferença entre os tempos. Ao observar os resultados, nota-se que há uma tendência a diferença do tempo 2 para o tempo $3(p=.09)$. 
Conforme os resultados apresentados pelo presente estudo, o treinamento em plataforma vibratória com duração de 6 semanas não foi capaz de alterar significativamente os índices de composição corporal dos sujeitos conforme os resultados apresentados (FIGURA 2).

Não foi possível notar mudanças consideráveis na perda de peso, aumento da massa muscular e na redução da massa de gordura, nos grupos GE e GV. Tais resultados podem se dar ao fato de que possivelmente o tempo da intervenção (6 semanas), juntamente com ausência do controle alimentar e o nível da intensidade dos exercícios, não foram capazes de produzir efeitos estatisticamente significativos. 0 exercício aeróbio adotado no protocolo deste estudo (GE) teve a intensidade adotada entre 4 e 6 na escala adaptada de Borg, e a frequência cardíaca (FC) entre $65-75 \%$ da FCM, valores considerados moderados conforme protocolos adotados.

Mezzaroba, Ribeiro e Machado (2014), Neves, Martins, Souza e Junior (2015), Teixeira Motoyama e Gentil (2016), e Capra et. al. (2016) afirmam que, durante muito tempo, se acreditou que o mais indicado para o processo de emagrecimento seria a utilização de exercícios de baixa intensidade, pois mobilizaria a gordura como principal substrato energético. Porém quando se pensa em modificar a composição corporal, evidências apontam que a combinação de exercícios físicos mais intensos acompanhados de um planejamento alimentar, tem demonstrado mais efetividade neste quesito

O exercício aeróbio foi durante muito tempo considerado a atividade mais recomendada no combate a redução do peso corporal, melhoras nas condições cardiorrespiratórias, aumento da tonicidade muscular e auxilio no tratamento e prevenção de doenças crônicas não degenerativas como a diabetes e hipertensão. Mas a sua intensidade, e o melhor protocolo a ser aplicado, merecem atenção, além de serem fatores de influência para o resultado final, pois é sabido que a realização de atividades aeróbias de intensidade moderada aumentam a mobilização de gorduras no momento do exercício, porém, atividades de alta intensidade, mobilizam mais ainda esse substrato no período pós-exercício (Alkahtani et al., 2013; Brooke-Wavell \& Mansfield, 2009; Huang et al., 2014; Júnior et al., 2011 Moezy et al., 2008; Osawa \& Oguma, 2013; Rubio-Arias et al., 2015; Santiago et al., 2015).

Já em relação ao GV, o presente estudo manteve e utilizou durante as sessões de treinamento, a mesma percepção subjetiva de esforço, na escala adaptada de Borg. Com os resultados também não apresentando diferenças significativas.

Diferentes protocolos têm sido testados por pesquisadores utilizando vibração. Desde reabilitação até a utilização de treinamento em plataformas vibratórias com a inserção de exercícios estáticos e dinâmicos. Dentre os estudos utilizando Treinamento de Vibração de Corpo Inteiro (WBVT), os resultados apresentaram uma relação positiva em outra variável: a densidade mineral óssea (DMO), e resposta neuromuscular para populações de idosos e mulheres pós-menopausa. Em adultos jovens, demonstrou que houve um aumento de baixo a moderado da ativação dos músculos do tronco durante os exercícios (Collado, Cristi-Montero, \& Cuevas,
Analisando a composição corporal em segmentos (tronco, perna direita e perna esquerda) após seis semanas de treinamento em plataforma vibratória, Rubios-Arias et al. (2015) demonstraram em seus estudos que não houve resultados significativos para um grupo de adultos jovens. Os autores concluíram que a estimulação produzida pela plataforma parece não ter sido suficiente para apresentar um alto índice metabólico e nem redução da massa gorda, fato que pode corroborar com o presente estudo.

Esta pesquisa ao comparar os exercícios de vibração com o exercício aeróbio numa população de adultos jovens sedentários, teve como intenção também, promover alternativas afim de firmar a adesão de uma atividade física no cotidiano deste publico. Assim como afirma Hallal et al. (2010), os protocolos utilizados nos exercícios de vibração são de curta duração e possui diferentes formas de utilização e combinações.

Apesar de não fazer parte desta investigação, foi encontrado que a junção de outras atividades ao método de treinamento em plataforma, já são realidade. A combinação vibração e treinamento resistido, parecem estar demonstrando melhores resultados comparando com o treinamento em plataforma sozinho. Um estudo realizado mostra a hipertrofia dos músculos do core através de Imagem de Ressonância Magnética (MRI) após treze semanas em dois grupos: treinamento resistido combinado com plataforma vibratória (RT-WBV) e somente o treinamento resistido (RT). Analisando a área da secção transversal, os autores verificaram uma diferença significativa nos valores pré e pós na hipertrofia dos músculos Iliopsoas e eretores da coluna, sendo que o maior valor de desenvolvimento muscular pode ser observado no grupo RT-WBV (Moezy et al., 2008; Rocha \& Snitynski, 2016; Teixeira et al., 2016).

Em nossa análise esbarramos na dificuldade da insuficiência de material para comparação com o tema desta pesquisa, ou seja, estudos com adultos jovens e a relação com a mudança na composição corporal e o treinamento em plataforma vibratória, o que justifica o uso de estudos com populações heterogenias para comparação em nossa pesquisa. Em resumo, 0 treinamento isolado com plataforma vibratória parece não ser tão eficiente quando combinado com o treinamento aeróbio ou resistido, apesar disto, são necessários mais estudos na área.

\section{CONCLUSÃO}

Ao final do estudo, pôde-se concluir que o treinamento em plataforma vibratória não apresentou resultados significativos que pudessem causar uma variação na composição corporal e densidade mineral óssea (DMO), em indivíduos adultos jovens e sedentários, porém ao longo da pesquisa, percebeu-se leves alterações que nos induz a crer que aumentando o volume e a intensidade desta atividade, os resultados podem ser positivos.

Dessa forma sugerem-se outros estudos controlando as variáveis de intensidade e volume de treino, amostras maiores e um controle alimentar. 
Aos representantes da Power Plate Brasil e a colaboração técnica dos funcionários no Laboratório de Avaliação Física e Treinamento (LAFIT) da Universidade Católica de Brasília (UCB).

\section{FONTES DE FINANCIAMENTO}

Conselho Nacional de Desenvolvimento Cientifico e Tecnologico -CNPq / processo no 446400/2014-9.

ANEXO 1. Organização do Programa de Treinamento na Plataforma Vibratória

\section{SEMANA 00}

NAMENTO NA PLATAFORMA VIBRATÓRIA

FAMILIARIZAÇÃO

\begin{tabular}{|c|c|c|c|c|c|c|c|}
\hline 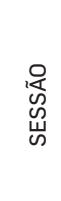 & 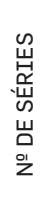 & 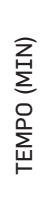 & 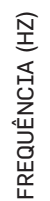 & 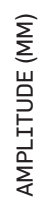 & 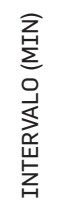 & 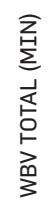 & \\
\hline
\end{tabular}

COLETA T1

\begin{tabular}{|c|c|c|c|c|c|c|c|c|}
\hline \multirow{3}{*}{ Semana 01} & 1 & 3 & 5 & \multirow{3}{*}{30} & \multirow{3}{*}{2} & 2 & 15 & \multirow{3}{*}{ Estático } \\
\hline & 2 & 2 & 7,30 & & & 2 & 15 & \\
\hline & 3 & 1 & 15 & & & - & 15 & \\
\hline \multirow{3}{*}{ Semana 02} & 4 & 3 & 5 & \multirow{3}{*}{30} & \multirow{3}{*}{4} & 2 & 15 & Dinâmico 1 \\
\hline & 5 & 2 & 7 & & & 2 & 15 & Dinâmico 2 \\
\hline & 6 & 1 & 15 & & & - & 15 & Dinâmico 3 \\
\hline \multirow{3}{*}{ Semana 03} & 7 & 3 & 5 & \multirow{3}{*}{35} & \multirow{3}{*}{2} & 2 & 15 & \multirow{3}{*}{ Estático } \\
\hline & 8 & 2 & 7,30 & & & 2 & 15 & \\
\hline & 9 & 1 & 15 & & & - & 15 & \\
\hline \multirow{3}{*}{ Semana 04} & 10 & 3 & 5 & \multirow{3}{*}{35} & \multirow{3}{*}{4} & 2 & 15 & Dinâmico 1 \\
\hline & 11 & 2 & 7,30 & & & 2 & 15 & Dinâmico 2 \\
\hline & 12 & 1 & 15 & & & - & 15 & Dinâmico 3 \\
\hline \multirow{3}{*}{ Semana 05} & 13 & 3 & 5 & \multirow{3}{*}{40} & \multirow{3}{*}{2} & 2 & 15 & \multirow{3}{*}{ Estático } \\
\hline & 14 & 2 & 7,30 & & & 2 & 15 & \\
\hline & 15 & 1 & 15 & & & - & 15 & \\
\hline \multirow{3}{*}{ Semana 06} & 16 & 3 & 5 & \multirow{3}{*}{40} & \multirow{3}{*}{4} & 2 & 15 & Dinâmico 1 \\
\hline & 17 & 2 & 7,30 & & & 2 & 15 & Dinâmico 2 \\
\hline & 18 & 1 & 15 & & & - & 15 & Dinâmico 3 \\
\hline
\end{tabular}

FASE

DE INTERVENÇÃo

COLETA T2

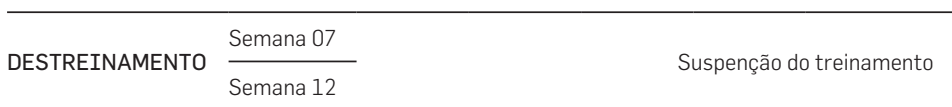

\section{COLETA T3}

Estático: em pé sobre a plataforma com os joelhos semi-flexionados com angulação de $30^{\circ}$ graus na articulação do joelho. A posição de estimulação será padronizada colocando o plano sagital do corpo sobre o ponto marcado no centro da base da plataforma vibratória e alinhando os pés à mesma distância dos ombros, descalços ou utilizando somente meias, com os membros superiores estendidos ao longo do tronco segurando em alças laterais que transmitem a vibraçăa também para os membros superiores. Dinâmico 1: Membro superior: flexão de cotovelo e abdução de ombro com cabo da plataforma ( $1 \times 10$ alternando os segmentos durante o tempo de exposição). Dinâmico 2: Membro Inferior: realização de agachamento até a angulação de 90 na articulação do joelho, $1 \times 10$ a cada 1' e recuperação passiva sem descer da plataforma, realização em velocidade lenta durante todo tempo de exposição. Dinâmico 3: Combinação do Dinâmico 1 e 2 (1x10 alternando os segmentos durante o tempo de exposição). Obs: O nivel da bolsa de ar será ajustado de acordo com as orientaç̄oes técnicas contidas no manual do fabricante.
Alkahtani, S. A., King, N. A., Hills, A. P., \& Byrne, N. Moezy, A., Olyaei, G., Hadian, M., Razi, M., \& Faghihzadeh, S. M. (2013). Effect of interval training intensity on ed exertion in obese men. SpringerPlus, 2(1), 532 . doi:10.1186/2193-1801-2-532

Brooke-Wavell, K., \& Mansfield, N. J. (2009). Risk and benefits of whole body vibration training in older people. Age and Ageing, 38(3), 254-255.

Capra, D., Tartaro, L. G., Magalhães, R. A., \& Martelli, A. (2016). Influência do treinamento de força em programas de em grecimento. Archives of Health Investigation, 5(1), 20.

Collado, P. S., Cristi-Montero. C., \& Cuevas, M. (2013). Whole-body vibration training as complement to programs aimed at weight loss. Nutrición Hospitalaria, 28(5), 1365-1371.

Gómez-Cabello, A., Gonzalez-Aquero, A., Ara, I., Casajús, J. A., \&Vicente-Rodriguez, G. (2013). Effects of a short-term whole body vibration intervention on physcal fitness in elderly people. Maturitas, 74(3), 276-278. cal uso da vibração de capacidas fiscas: Uma revisa da Uteratra. Motriz: Revista de Educação Física, 16(2), 527-533. Haskell, W. L., Lee, I. M., \& Pate, R. R. (2007). Physical activity and public health: updated recommendation for adults from the American College of Sports Medcine and the American Heart Association. Circulation 116(9), 1081-1093.

Huang, C. C.. Tseng. T. L., Huang, W. C.. Chung. Y. H., Chuang H. L., \& Wu, J. H. (2014). Whole-body vibration training effect on physical performance and obesity in mice. International Journal of Medical Sciences, 11(12), 1218-1227. Júnior, F. L. P., Prestes, J., Leite, R. D., \& Rodriguez, D. (2011). Influência do treinamento aeróbio nos mecanismos fisiopatológicos da hipertensão arterial sistêmica. Revista Brasileira de Ciências do Esporte, 32(2-4), 229-244.

iu, P. Y. Kenneth, B. S. \& Jasminka, Z. I (2011). Aerobic exercise and whole-body vibration in offsetting bone loss in older adults. Journal of Aging Research, 2011, 379674 Mendes, C. M. L., \& da Cunha, R. C. L. (2013). As nova tecnologias e suas influencias na prática de atividade física e no sedentarismo. Revista Interfaces: Saúd Humanas e Tecnologia, 2(1), 1-23.

Mezzaroba, P. V., Ribeiro, M. S., \& Machado, F. A. (2014). Comparação de dois métodos de treinamento contra resistência na força, antropometria e composição corporal de mulheres jovens. Revista Brasileiria de Ciência e Movimento, 22(2), 106-113. (2008). A comparative study of whole body vibration traning and conventional training on knee proprioception and postural stability after anterior cruciate ligament reconstruction. British Journal of Sports Medicine, 42(5), 373-385. Neves, D. R., Martins, E. A., Souza, M. V. C., \& Junior, A. J. S. (2015). Efeitos do treinamento de força sobre o índice do percentual de gordura corporal em adultos. Revista Brasileira de Obesiddde, Nutrição e Emagrecimento, 52(9), 135-141.

de Oliveira Belmiro, W \& Navarro, A. C. (2016). Os efeitos do treinamento intervalado de alta intensidade para o emagrecimento. Revista Brasileira de Obesidade, Nutrição e Emagrecimento, 59(10), 224-230. Osawa, Y., \& Oguma, Y. (2013). Effects of resistance training with whole-body vibration on muscle fitness in untrained adults. Scandinavian Journal of Medicine \& Science in Sports, 23(1), 84-95.

Rocha, C., \& Snitynski, D. (2016). 0 efeito do Hiit na composição corporal de mulheres sedentárias. Anais do EVINCI-UniBrasil, 165-165.

Rubio-Arias, J. A., Esteban, P., Martinez, F., RamosCampo, D. J., Mendizabal, S., Berdejo-del-Fresno, D., \& Jimenez-Diaz, J. F. (2015). Effect of 6 weeks of whole body vibration training on total and segmental body composition in healthy young adults. Acta Physiologica Hungarica, 102 (4), 442-450.

Santiago, L. A. M., Neto, L. G. L., Santana, P. V. A., Mendes, P. C., Lima, W. K. R., \& Navarro, F. (2015). Resisted training reduces cardiovascular risk in elderly women. $R e-$ vista Brasileira de Medicina do Esporte, 21(4), 261-265. Silva, P. Z., \& Schneider, R. H. (2011). Efeitos da plataforma vibratória no equilibrio em idosos. Acta fisiátrica, 18(1), 21-26. Silva, D. A. S., \& Nunes, H. E. G. (2015). 0 que é mais eficiente para perda de peso: Exercício contínuo ou intermitente? Com ou sem dieta? Uma revisão baseada em evidências Medcin (Riberio Preto Onlie) 48(2) 110-128. Teixeira, C. V. L. S., Motoyama, Y., \& Gentil, P. (2016). Musculação: Crenças vs. evidências. Revista Brasileira Verschueren, S. M., Roelants, M., Delecluse, C., Swinnen, S., Vanderschueren, D., \& Boonen, S. (2004). Efeito do treino de vibração de corpo inteiro durante 6 meses na densidade da anca, força muscular e controlo posturat das mutheres pós menopausa, Journol of Bone and Mineral Research, 19(3), 612-622.

Vieira, L. G. U., \& Queiroz, A. C. C. (2013). Análise metodológica do treinamento de força como estratégia de controle da pressão arterial em idosos: Uma revisão. Revisto Brasileira de Geriatria e Gerontologia, 16(4), 845-854. de Prescrição e Fisiologia do Exercício, 55(9), 562-571. 